\title{
User Parameter Tunning for VoD Service Users
}

\author{
A. Echavarren, C. Zubieta, J. Villadangos, and M. Prieto \\ Área de Ingeniería Telemática, Departamento de Automática y Computación. \\ Universidad Pública de Navarra Campus de Arrosadia, 31.006 Pamplona.
}

\begin{abstract}
This work has been focused on the design of a VoD parametermeasuring tool. This tool works as a video client and additionally provides a set of measurements used to define different user parameters in different network conditions.
\end{abstract}

\section{Introduction}

The operation of a VoD service involves a number of engineering decisions that affect the VoD quality of service. Some papers in the literature consider the system performance $[1,4]$, others, that the bottleneck of the $\mathrm{VoD}$ service is the I/O bandwidth $[1,2]$, and there are different proposals to optimise the video server performance by applying different scheduling algorithms to serve the movies $[2,3]$.

However, there are other parameters that should be taken into account both in the client as in the network side. The client usually uses a pre-load buffer to store the first bytes of the movie to avoid starvation during video playback and there is also a very important decision about the size of the playback buffer of the client player. In the same way, it is necessary to measure the effect of the network during the movie transmission.

\section{Measuring Tool Architecture}

The measuring tool has been developed based on the DirectX graph filter architecture for Windows [5]. It provides a flexible and adaptive tool as it can works with all the registered filters in Windows.

The measuring tool consists on a filter graph built by the filter manager (see figure 1). An asynchronous input filter has been developed ad-hoc to provide the measuring tool characteristics (see figure 1). The other necessary filters are the same to those needed to play the file from the hard disc.

The asynchronous input filter manages the data transmission between the video server and the player. The received data from the network is stored in a circular buffer and when the next filter asks an amount of data the developed input filter gives the data from the circular buffer. Consequently, DirectShow is used to make the necessary 
conversions and to render the samples correctly. In addition, the input filter allows monitoring parameters as the bytes/s, packets/s, and so on. In addition, it provides the graphical representation of the parameters. Moreover, the developed input filter manages different possible critical circumstances (see figure $1 \mathrm{a}, \mathrm{b}$ ).

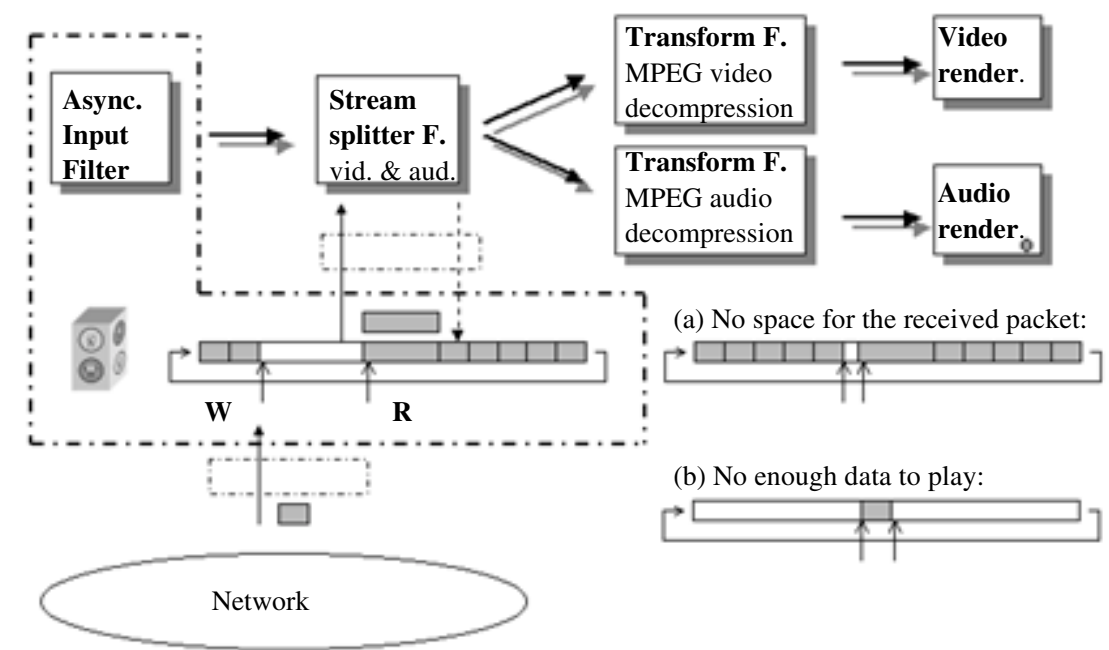

Fig. 1. A filter graph built in the video client to play an MPEG video file

\section{Conclusions}

This experience has shown from the point of view of the client that many points have to be considered in a VoD service. The developed tool allows setting different user parameters and evaluating the VoD service.

\section{References}

1. Sudhanshu Aggarwal and Juan A. Garay and Amir Herzberg. Adaptive video on demand. Proc of the 13th annual ACM symposium on Principles of distributed computing, 1994

2. Min-You Wu, Su-Jun Ma, Wei Shu. Scheduled video delivery for scalable on-demand service Proc of the 12th International Workshop on Network and Operating Systems Support for Digital Audio and Video, 2002

3. Emmanuel L. Abram-Profeta and Kang G. Shin. Scheduling video programs in near videoon-demand systems. Proc of the fifth ACM international conference on Multimedia, 1997

4. N. Judice, E. J. Addeo, M. I. Eiger, H. L. Lemberg. Video on Demand: A Wideband Service or Myth? Proc. of ICC'86, June 1986

5. Use Multimedia Streaming in DirectShow Applications. MSDN Library January 1999. Microsoft Corporation. 